\title{
Evaluation of the nasal contractility capacity in postmenopausal women*
}

\author{
Yavuz Selim Yıldırım, Erol Senturk, Selahattin Tugrul, Orhan Ozturan \\ Bezmialem Vakif University, Department of Otorhinolaryngology, Fatih, Istanbul, Turkey
}

Rhinology 52: 397-402, 2014

DOI:10.4193/Rhino13.107

*Received for publication:

July 22, 2013

Accepted: February 16, 2014

\begin{abstract}
Objective: The aim of this study was to objectively compare nasal contractility in premenopausal and postmenopausal patients by means of rhinomanometry, acoustic rhinometry, and peak inspiratory nasal flow.
\end{abstract}

Methods: Design: prospective, clinical case - controlled study. Setting: university tertiary-care hospital, referral center. Participants: the study included 97 participants divided into two groups: 49 postmenopausal (the study group) and 48 premenopausal (control) females. Nasal evaluations were made utilizing anterior rhinoscopy, peak inspiratory nasal flow, acoustic rhinometry, and anterior rhinomanometry.

Results: The differences between before and after decongestant application of Minimal Cross-sectional Area (MCA) 1, Minimal Cross-sectional Area (MCA) 2, Peak inspiratory nasal flow (PNIF), Rhinomanometry (RMM), Flow, and Volume values were been statistically significant in both postmenopausal and premenopausal group (control).

Conclusion: Before and after all the subjects were administered nasal decongestant, nasal contractility was evaluated using objective nasal tests (acoustic rhinometry, rhinomamometry, and peak inspiratory nasal flow. Results showed that erectile tissues were not affected after menopause: postmenopausal women have the same nasal contractility capacity as premenopausal women.

Key words: aging, menopause, acoustic rhinometry, rhinomanometry, postmenopause, concha

\section{Introduction}

Nasal mucosa is under hormonal control, and various nasal symptoms have been described in association with hormonal changes. In postmenopausal women, low oestrogen and progesterone levels mean that the ratio of oestrogen and progesterone to androgens becomes more important. In the postmenopause stage, insufficient oestrogens cause women to complain of reduced nasal function and nasal blockage ${ }^{(1)}$. Nasal obstruction can be caused by a variety of factors. The subjective sensation of nasal obstruction can be misleading, thus objective methods should be used to evaluate the obstructions of the nose. The most commonly used objective methods are rhinomanometry and acoustic rhinometry. Rhinomanometry provides dynamic data on nasal function by measuring resistance of nasal airflow. Acoustic rhinometry gives anatomical information about the site of the obstruction (2).

Since the effect of menopause on nasal mucosa is still a matter of controversy, it is not surprising that the potential mechanisms that may account for possible differences are highly speculative. Popovic et al. ${ }^{(3)}$ have suggested that oestrogen and progesterone exert a protective effect on the upper airway.

The nose goes through the inevitable process of aging, yet in 
comparison to the many published reports on the aging process of the body's other organs and tissues, little attention has been paid to the nose. Of those, most concern nasal change in the epithelial, olfaction, or taste. There are few studies in the literature on changes of the nasal cavity, and none on the relationship between aging and contractility capacity ${ }^{(4)}$.

The purpose of this study was to compare the nasal contractility capacity in premenopausal and postmenopausal women in a healthy population.

\section{Materials and methods}

The 97 participants were divided into the study (postmenopausal) group ( $\mathrm{n}=49 ;>64$ years) and the control (premenopausal) group ( $n=48$; aged 20 - 40 years). The median ages were 66.44 years (range: 64 - 82 years) and 29.2 years (range: 20 - 40 years), respectively. All participants underwent an otorhino-laryngological examination including anterior rhinoscopy and a 0 degree nasal endoscopy without application of decongestants or local anesthesia. Exclusion criteria included sinonasal symptoms, prior sinonasal surgery or deviated nasal septum, septum perforation, allergic rhinitis or other rhinitis, other sinonasal diseases or problems, upper respiratory infection, a sleep-related disorder, use of drugs (e.g., antihistamines or decongestants, diuretics, antihypertensives, and antidepressants), or drug medication in the previous one month. The study and control subjects were healthy volunteers enlisted from among patients with nonrhinologic complaints or hospital employees. The institutional research review board of the hospital approved the study and informed consent was received from all subjects.

After 20 minutes of acclimatization, acoustic rhinometry and rhinomanometry was performed without nasal vasoconstriction. Nasal decongestant was applied with two puffs of xylometazoline spray (lliadin nasal spray $0.05 \%$ ) into each nostril and, 15 minutes later, peak inspiratory nasal flow, rhinometry, and rhinomanometry was measured again. The room temperature and humidity were kept constant by means of central air conditioning and a humidifier.

Peak inspiratory nasal flow rate measurement (PNIFR) Peak inspiratory nasal flow rate measurement (PNIFR) was performed using a Youlten flowmeter (Clement Clarke International, London, UK). Each subject was given three opportunities to record maximum inspiratory flow rates $(\mathrm{mL} / \mathrm{min})$ and the two lower values were discarded. This method has previously been shown to be an objective measurement of nasal patency ${ }^{(5)}$.

\section{Acoustic rhinometry (AR)}

An acoustic rhinometer (NR-6, GM Instruments Ltd., Kilwinning, UK) was used for acoustic rhinometry. Subjects were seated upright in a straight-backed chair facing straight ahead and were tested three times per nostril. A well-fitted nose tip made an acoustically tight seal between the nostril and the nose tip. The mean MCA and standard deviation were measured by the computer software (Naris software). The minimum MCAs at the first and second valley of the AR graph were designated as MCA 1 and MCA 2, respectively. The MCAs of the left and right side were added and analyzed with reference to age.

\section{Rhinomanometry (RMM)}

A rhinomanometer (A-1, GM Instruments Ltd., Kilwinnig, UK), a kind of active anterior rhinomanometry, was used to measure air pressure and flow. Subjects were instructed to breathe through the adaptor that was connected to the manometer. Rhinomanometry was performed on both sides of the nose and flow and pressure measurements were performed at rest and then 15 minutes after application of a xylometazoline spray. Pressure at 150 pascal was used for analysis and total resistance was calculated using the following equation: Total Resistance1/ (1/RRight1/Rleft). Total resistance and flow were analyzed with reference to age.

\section{Statistical analyses}

The normality of the differences between baseline and other measurements of acoustic values, nasal resistance, and VAS variables were evaluated with the Shapiro-Wilk test. Student t-test was used for intergroup comparisons, and a one-way analysis of variance was used for multigroup comparisons. In case of statistical significance, post hoc test (Duncan's method) was applied to identify any difference between age groups. For groups with a normal distribution and a $95 \%$ confidence interval with an $a=$ 0.05 and a $1-\beta=0.80, p<0.05$ was deemed to indicate statistical significance. All analyses were performed by the MedCalc (version 12.3.0; MedCalc Software, Turkey) and Windows SPSS (version 16.0.1; SPSS, Inc., Chicago, IL, USA) statistical packages.

\section{Results}

A significant difference was observed between the two groups in terms of age $(p<0.0001)$. Demographic and statistical data for the study and control groups are summarized in Tables 1 and 2.

Before and after application of decongestants to patients in the postmenopausal group, the differences of MCA 1, MCA 2, PNIF, RMM, and volume values were statistically significant $(p<0.005)$, but no significant relationship was observed in nasal flow $(p=$ 0.274).

Before and after application of decongestion the premenopausal (control) group, the differences of MCA 1, MCA 2, PNIF, RMM, nasal flow, and volume values were all statistically significant $(p$ $<0.005)$. 
Table 1. Summary statistics table for the menopause group.

\begin{tabular}{lccccccccc}
\hline Menopause group & N & Mean & $95 \%$ CI & Variance & SD & RSD & Median & Min & Max \\
\hline Age & 49 & 66,44 & $64,6-68,2$ & 37,91 & 6,15 & 0,09 & 65,0 & 55,0 & 82,0 \\
\hline MCA 1 With D & 49 & 1,01 & $0,9-1,0$ & 0,065 & 0,25 & 0,25 & 0,9 & 0,4 & 1,7 \\
\hline MCA 1 Without D & 49 & 0,79 & $0,73-0,85$ & 0,040 & 0,20 & 0,25 & 0,8 & 0,3 & 1,3 \\
\hline MCA 2 With D & 49 & 3,43 & $3,0-3,7$ & 1,51 & 1,23 & 0,35 & 3,2 & 0,8 & 6,8 \\
\hline MCA 2 Without D & 49 & 2,44 & $2,2-2,6$ & 0,53 & 0,73 & 0,30 & 2,5 & 1,0 & 4,7 \\
\hline RMM With D & 49 & 0,22 & $0,1-0,3$ & 0,01 & 0,12 & 0,54 & 0,1 & 0,1 & 0,4 \\
\hline RMM Without D & 49 & 0,23 & $0,21-0,26$ & 0,008 & 0,09 & 0,38 & 0,2 & 0,1 & 0,5 \\
\hline Volume With D & 49 & 11,09 & $10,0-12,1$ & 12,58 & 3,54 & 0,31 & 10,3 & 4,7 & 20,7 \\
\hline Volume Without D & 49 & 8,85 & $8,0-9,68$ & 8,34 & 2,88 & 0,32 & 8,1 & 4,2 & 18,37 \\
\hline PNIF With D & 49 & 135,71 & $96,1-175,2$ & 1828,57 & 42,76 & 0,31 & 140,0 & 70,0 & 200,0 \\
\hline PNIF Without D & 49 & 109,18 & $101,1-117,1$ & 778,48 & 27,90 & 0,25 & 100,0 & 50,0 & 180,0 \\
\hline FlowWith D & 49 & 810,42 & $503,7-1117,0$ & 109942,95 & 331,57 & 0,40 & 954,0 & 324,0 & 1169,0 \\
\hline FlowWithout D & 49 & 706,83 & $644,5-769,1$ & 47013,88 & 216,82 & 0,30 & 689,0 & 293,0 & 1115,0
\end{tabular}

$M C A=$ minimal cross-sectional area $\left(\mathrm{cm}^{2}\right) ; \mathrm{VOL}=$ volume $0-7 \mathrm{~cm}$ from the nostril $\left(\mathrm{cm}^{3}\right) ; \mathrm{RMM}=$ nasal resistance $(\mathrm{Pa} / \mathrm{mL} / \mathrm{s}) ; \mathrm{PNIFR}$ : peak nasal inspiratory flow rate

Table 2. Summary statistics table for the control group.

\begin{tabular}{lccccccccc} 
Control group & N & Mean & 95\% CI & Variance & SD & RSD & Median & Min & Max \\
\hline Age & 48 & 29,2 & $27,1-31,2$ & 42,52 & 6,52 & 0,2 & 28,0 & 20,0 & 40,00 \\
\hline MCA 1 With D & 48 & 0,9 & $0,8-1,0$ & 0,13 & 0,36 & 0,3 & 0,9 & 0,5 & 1,80 \\
\hline MCA 1 Without D & 48 & 0,6 & $0,5-0,7$ & 0,05 & 0,24 & 0,3 & 0,5 & 0,2 & 1,21 \\
\hline MCA 2 With D & 48 & 3,1 & $2,8-3,4$ & 0,93 & 0,96 & 0,3 & 3,2 & 1,5 & 5,53 \\
\hline MCA 2 Without D & 48 & 1,8 & $1,6-2,0$ & 0,42 & 0,64 & 0,3 & 1,8 & 0,5 & 4,14 \\
\hline RMM With D & 48 & 0,1 & $0,13-0,19$ & 0,001 & 0,03 & 0,1 & 0,1 & 0,1 & 0,20 \\
\hline RMM Without D & 48 & 0,2 & $0,2-0,3$ & 0,01 & 0,11 & 0,4 & 0,2 & 0,1 & 0,73 \\
\hline Volume With D & 48 & 10,6 & $9,7-11,5$ & 7,42 & 2,72 & 0,2 & 9,9 & 6,3 & 18,28 \\
\hline Volume Without D & 48 & 7,5 & $6,9-8,1$ & 3,56 & 1,88 & 0,2 & 7,2 & 4,6 & 12,87 \\
\hline PNIF With D & 48 & 152,8 & $127,4-178,3$ & 757,14 & 27,51 & 0,18 & 160,0 & 100,0 & 180,00 \\
\hline PNIF Without D & 48 & 117,7 & $109,9-125,6$ & 602,50 & 24,54 & 0,2 & 110,0 & 70,0 & 170,00 \\
\hline FlowWith D & 48 & 919,2 & $744,99-1093,5$ & 35516,23 & 188,45 & 0,2 & 826,0 & 720,0 & 1218,00 \\
\hline FlowWithout D & 48 & 629,8 & $562,2-697,4$ & 44641,51 & 211,28 & 0,33 & 631,0 & 204,0 & 1169,00
\end{tabular}

MCA = minimal cross-sectional area $\left(\mathrm{cm}^{2}\right) ; \mathrm{VOL}=$ volume $0-7 \mathrm{~cm}$ from the nostril $\left(\mathrm{cm}^{3}\right) ; \mathrm{RMM}=$ nasal resistance $(\mathrm{Pa} / \mathrm{mL} / \mathrm{s}) ;$ PNIFR: peak nasal inspiratory flow rate 


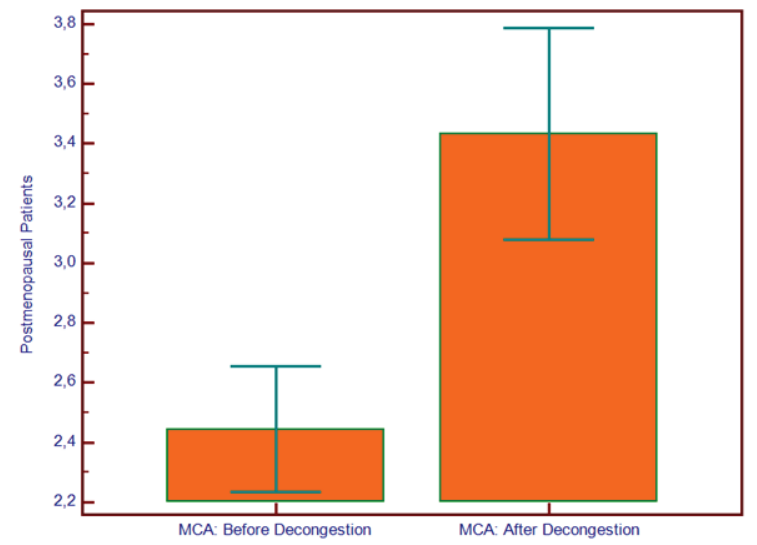

Figure 1. Minimal Cross-sectional Area (MCA) measured by Acoustic Rhinometry before and after decongestant showing a statistically significant increase of MCA values in postmenopausal patients $(p<0.005)$.

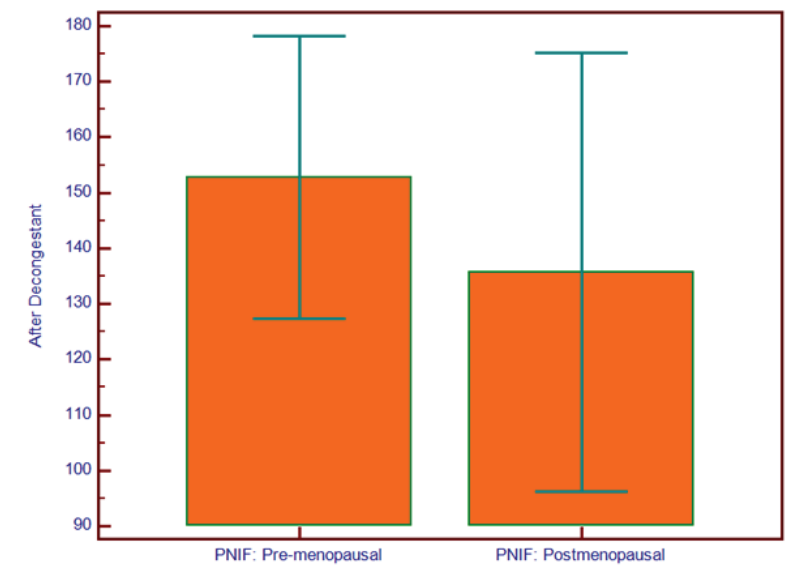

Figure 2. Peak inspiratory nasal flow rate (PNIF) measured after application of a decongestant. There are no statistically significant changes in the postmenopausal patients compared with the premenopausal patients $(p<0.39)$.

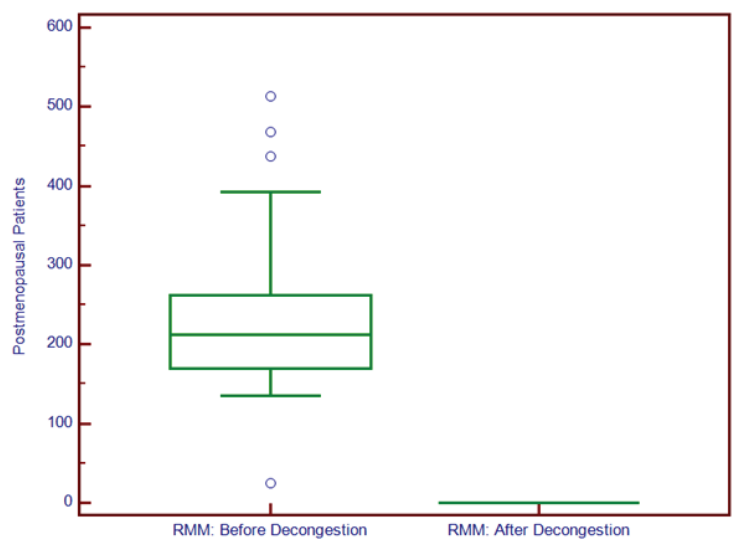

Figure 3. Nasal Resistance (RMM) measured by rhinomanometry after application of a decongestant showing significantly decreased RMM values in postmenopausal patients $(p<0.005)$.

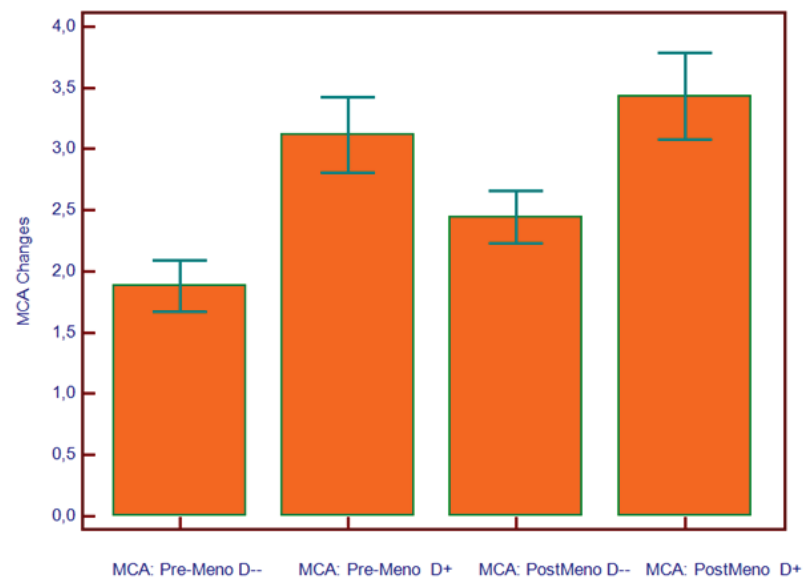

Figure 4. Minimal Cross-sectional Area (MCA) measured by Acoustic Rhinometry before and after application of a decongestant. The MCA value in postmenopausal patients shows no statistically significant relationship with the premenopausal patients $(p<0.49, p<0.19)$.
The differences of before-decongestion application MCA 1, MCA 2, RMM, and volume values were statistically, significantly higher in the premenopausal group compared with the postmenopausal group, but no statistically significant difference was established between PNIF and nasal flow values ( $p=0.0132$ and 0.09 ).

The differences of after-decongestion application MCA 1 ( $p=$ $0.49), \operatorname{MCA} 2(p=0.19)$, PNIF $(p=0.39), \operatorname{RMM}(p=0.25)$, flow $(p=$ $0.46)$, and volume, $(p=0.52)$ values were not statistically significant in either group.

\section{Discussion}

Similar to other organs of the body, the nose changes as an individual ages. The feeling of a dry nose, recurrent intranasal crusting, postnasal dripping, and obstructed nasal breathing are some of the common reasons for elderly people to present at an ENT outpatient clinic. Several approaches exist to explain these particular rhinological problems. Older patients are supposed to normally present reduced nasal mucosal sensitivity ${ }^{(6)}$. In older people, the generalized decrease in body fluids leads both to dryness of nasal mucosa and increased viscosity of mucus secretion, associated with degeneration and atrophy of mucus-secreting glands and loss of lymphatic tissue. This further decreases moisture content, reducing the effectiveness of the nasal function and leading to symptoms of nasal stuffiness ${ }^{(7)}$. Our study investigated the changes in the acoustic rhinometry, anterior rhinomanometry, and peak inspiratory nasal flow of postmenopausal women compared with premenopausal wo- 
men, before and after application of a decongestant. We were unable to detect any statistically significant differences between the two groups in terms of nasal contractility capacity.

The sex hormones have a possible mediating effect through histamine receptors by increasing the expression of $\mathrm{H} 1$ receptors in the nasal epithelium and microvascular endothelial cells ${ }^{(8)}$. Bowser et al. ${ }^{\left({ }^{9}\right)}$ suggested that fibroblasts in the nasal mucosa of pregnant women are influenced by progesterone, but that oestrogen receptor staining observed in turbinate tissue was nonspecific. They also proposed that oestrogens and progesterone change neurotransmitter concentrations such as substance $P$ cause the symptoms of nasal stuffiness experienced by women during pregnancy ${ }^{(10)}$.

The influence of endogenous hormones upon nasal physiology, menstrual cycles, and during pregnancy has been shown in previous studies ${ }^{(11)}$. One randomized, double-blind, placebocontrolled study demonstrated that a sequential continuous regimen of hormone therapy (transdermal 17- $\beta$-estradiol plus nomegestrole acetate) exerts a positive effect on nasal function in postmenopausal women complaining of paradoxical nasal stuffiness (i.e., sensation of nasal blockage without swelling or anatomical alterations). They also demonstrated that oestrogens modulate nasal mucosal function through an action on cholinergic, adrenergic, and sensory peptides ${ }^{(12)}$. However, when these hormones are applied in a non-physiological and endogenous manner, this effect is not seen ${ }^{(13)}$.

A study of postmenopausal women undergoing a trial of nasal versus systemic hormone replacement therapy recruited subjects to have their nasal physiological parameters measured before and during treatment, and still no significant effect was found on nasal physiology ${ }^{(14)}$. A similar study looking at the same parameters before and after onset of a combined oral contraceptive also showed no effect ${ }^{(13)}$.

A study that looked at the concentration of female hormone receptors in the nasal mucosa has shown that oestrogen $\beta$ receptors were found in 25 of 26 female nasal mucosal samples, whereas progesterone and oestrogen a receptors were not ${ }^{(14)}$. This finding further helps us to understand the exact mechanism of the action of female sex hormones on nasal physiology. In one study, 20 postmenopausal women underwent assessment of their nasal airways before and after onset of female hormone replacement therapy (HRT). It found that female HRT has no discernible effect upon nasal physiology and thus should not be considered a cause of rhinitis symptoms ${ }^{(15)}$.

Histochemical investigation of menopausal females found an increase in carbohydrate metabolism, lipid breakdown, phago- cytic activity, vascularity, secretory activity, and parasympathetic hyperactivity. These changes were not related to ovarian steroid hormones ${ }^{(16)}$. During the postmenopausal period, nasal function is important because application of intranasal calcitonin, progesterone, and oestrogen spray are means of administering hormone replacement therapy.

Results of this study showed that nasal resistance (RMM) and cross sectional area (CSA) were not significant with postmenopausal patients compared to premenopausal patients in the concha contractility. Reduced nasal function and nasal blockage can be attributed to age and individual traits in the postmenopausal period.

Our findings could be limited to potential conditioning for the patient doing a test (e.g., PNIF). We were able to compare postmenopausal and premenopausal women eligible for follow-up and found statistically significant differences in menopausal status, but we are not taking into account body mass index. In additon, we have not performed mucociliary clearance, because the mucociliary system may be age-dependent and may be confounded by this study.

Although MCA increased with increasing age, the difference in MCA before and after decongestion was not significant. Atrophy of bone or other structural parts of the nasal cavity with increasing age could be one of the causes, since mucosal shrinkage did not change with age. In other words, even though the nasal cavity area increased, increasing age did not coincide with any observed differences in mucosal shrinkage ${ }^{(4)}$.

In summary, this study used objective nasal tests (acoustic rhinometry, rhinomanometry, and peak inspiratory nasal flow) to evaluate nasal contractility before and after application of a nasal decongestant on premenopausal and postmenopausal subjects. We found that erectile tissues were not affected after menopause: postmenopausal women have the same nasal contractility capacity as premenopausal women, which means shrinkage of the erectile tissues did not change. It could be concluded from these findings that erectile tissues were not affected by postmenopausal changes.

\section{Authorship contribution}

YSY: drafting the article, analysis and interpretation of data

ES: drafting the article, collecting data

ST: content and analysis and interpretation of data

OO: revising it critically for important intellectual content

\section{Conflict of interest}

No funding source, financial disclosures, or sponsor. 


\section{References}

1. D'haeseleer E, Depypere H, Claeys S, Van Lierde K. The impact of menopause and hormone therapy on nasal resonance. Logoped Phoniatr Vocol. 2012; 3: 69-74.

2. Clement PAR, Gordts F. Consensus report on acoustic rhinometry and rhinomanometry. Rhinology. 2005; 43: 169-179.

3. Popovic RM, White DP. Upper airway muscle activity in normal women: influence of hormonal status. J Appl Physiol. 1998; 84: 1055-1062.

4. Kim SW, Mo JH, Kim JW, et al. Change of nasal function with aging in Korean. Acta Otolaryngol Suppl. 2007; 558: 90-94.

5. Fairley JW, Durham LH, Ell SR. Correlation subjective sensation of nasal patency with nasal inspiratory peak flow rate. Clin Otolaryngol. 1993; 18: 19-22.

6. Lindemann J, Sannwald D, Wiesmiller K. Age-related changes in intranasal air conditioning in the elderly. Laryngoscope. 2008; 118: 1472-1475.

7. Janzen VD. Rhinological disorders in the elderly. J Otolaryngol 1986; 15: 228-230.

8. Mabry RL. The management of nasal obstruction during pregnancy. Ear Nose Throat J. 1983: 62: 28-33.
9. Bowser C, Riederer A. Detection of proges terone receptors in connective tissue cells of the lower nasal turbinates in women. Laryngo-rhino-otol. 2001; 80: 182-186.

10. Bende M, Hallgarde U, Sjogren U, et al Nasal congestion during pregnancy. Clin Otolaryngol. 1989.14: 385-387.

11. Philpott CM, Conboy P, Al-Azzawi F, Murty GE. Nasal physiological changes during pregnancy. Clin Otolaryngol. 2004; 29: 343351.

12. Nappi C, Di Spiezio Sardo A, Guerra G, Bifulco G, Testa D, Di Carlo C. Functional and morphological evaluation of the nasal mucosa before and after hormone replacement therapy in postmenopausal women with nasal complaints. Fertil Steril. 2003; 80: 669-671.

13. Wolstenholme CR, Philpott CM, Oloto EJ Murty GE. Does the use of the combined oral contraceptive pill cause changes in the nasal physiology in young women? Am J Rhinol. 2006; 20: 238-240.

14. Wolstenholme CR, Wild DC, and Murty GE. Assessment of the effects of hormone replacement therapy in post-menopausal women on nasal physiology. Otolaryngol Head Neck Surg. 2005; 133: 155.
15. Wild DC, Philpott CM, Wolstenholme CR, Murty GE. Does hormone replacement therapy in post-menopausal women have any effect upon nasal physiology? Laryngol Otol. 2008; 122: 707-710.

16. Toppozada $H$. The human nasal mucosa in the menopause: A histochemical and electron microscopic study. J Laryngol Otol. 1988: 102; 314-318.

Yavuz Selim Yıldırım, M.D

FEBORL-HNS

Bezmialem Vakif University

Medical Faculty

Department of Otorhinolaryngology,

Fatih, Istanbul

Turkey

Tel: +90 $536216-1622$

Fax: +90 212 533-2326

E-mail: dryavuzselim@yahoo.com 